\title{
CASE REPORT \\ Characterization of spinal subarachnoid bleeding associated to graded traumatic spinal cord injury in the rat
}

\author{
HJ Reyes-Alva ${ }^{1,3}$, RE Franco-Bourland ${ }^{2,4}$, A Martinez-Cruz ${ }^{2}$, I Grijalva ${ }^{1,2}$, I Madrazo ${ }^{1,2}$ \\ and G Guizar-Sahagun ${ }^{1,2}$
}

Study design: Observational study in rats subjected to traumatic spinal cord injury ( $\mathrm{SCl}$ ).

Objectives: To describe the features of spinal subarachnoid bleeding (SSB) occurring after graded SCI. SSB after SCI has been reported previously, but has not been studied systematically despite the fact that cerebral subarachnoid bleeding often produces severe neurological damage.

Setting: Mexico.

Methods: Anesthetized rats were subjected to mild or severe spinal cord contusion at T9. Occurrence, size, progression and location of SSB were characterized morphologically and scored from T7-T12 at $1 \mathrm{~h}$ and 1,3 and 7 days post injury. Besides, contusions were videotaped to visualize bleeding at the moment of impact.

Results: SSB started immediately after contusion (severe or mild) and decreased gradually over time. For all vertebral segments, at all time points examined by histology, $48 \%$ of areas scored after severe contusion showed bleeding: $25 \%$ minor, $17 \%$ moderate and $6 \%$ major. After mild contusion, only 15\% showed bleeding: 13 minor and 2\% moderate. Maximum bleeding occurred early after injury in dorsal area of the epicenter in $100 \%$ of severe contusions ( $6 \%$ minor, 38 moderate and $56 \%$ major), and in $69 \%$ of mild contusions (63 minor and $6 \%$ moderate).

Conclusion: Here, we detail SSB patterns occurring after graded SCI. Further studies are warranted to elucidate the possible role extramedullary events, such as SSB, in the pathophysiology of SCI that might encourage the development of new strategies for its management.

Spinal Cord (2014) 52, S14-S17; doi:10.1038/sc.2014.93

\section{INTRODUCTION}

Spinal cord injury (SCI) produces severe and permanent neurological impairment, attributed to pathophysiological mechanisms whose treatment has not yielded results worth implementing clinically.,2 Hence, the importance of identifying novel pathomechanisms, possibly extramedullary events like spinal subarachnoid bleeding (SSB), that could become targets for new therapeutic strategies. SSB occurs after clinical ${ }^{3}$ and experimental ${ }^{4,5}$ traumatic SCI.

In a recent report, ${ }^{5}$ we describe the spinal subarachnoid space (SSAS) alterations that occur after graded spinal cord contusions. SSAS lumen is reduced at acute and subacute stages after injury. Notably, in severe cases, SSAS reduction and compression of neurovascular elements contained therein appear to be due to cord swelling and subarachnoid bleeding. Extramedullary events, such as these, may contribute to SCI pathophysiology by altering cerebrospinal fluid (CSF) dynamics and cord blood perfusion, which if addressed, might lead to a better outcome after cord injury.

Considering that extravasated subarachnoid blood in brain ${ }^{6,7}$ and spinal cord (not related to SCI), ${ }^{8}$ can result in severe neurological deterioration and even death, we designed the current study to establish the extent and time course of SSB in acute and subacute stages after graded SCI in a validated rat model that reproduces human cord injury. ${ }^{9}$

\section{MATERIALS AND METHODS}

Adult female Long-Evans rats (240-260 g) were subjected to graded spinal cord contusion. For histological analysis (incidence, size, progression and location of extravasated blood in SSAS), animals ( $n=8$ per group) were killed at $1 \mathrm{~h}$ and 1, 3 and 7 days post injury. Additional rats with graded injuries $(n=3$ per group) were killed at the same time points for macroscopic viewing of SSB. Videotaping of severe and mild contusions ( $n=3$ per group) was performed at the moment of injury to visualize very early bleeding.

\section{Anesthesia, injury and cares}

For cord injury, animals were anesthetized with ketamine $\left(80 \mathrm{mg} \mathrm{kg}^{-1}\right)$ and xylazine $\left(8 \mathrm{mg} \mathrm{kg}^{-1}\right)$ given intramuscular. A laminectomy was performed aseptically at $\mathrm{T}$. SCI was produced using the NYU impactor by dropping onto the exposed dura the rod weighing $10 \mathrm{~g}$ from a height of $12.5 \mathrm{~mm}$ for mild intensity injuries, and from $50 \mathrm{~mm}$ for severe injuries. Post-surgical care included manual expression of bladders twice a day. Food and water were provided ad libitum. Rats received ciprofloxacin lactate $\left(8 \mathrm{mg} \mathrm{kg}^{-1}\right.$ subcutaneously every $12 \mathrm{~h}$ ), and acetaminophen in the drinking water (approximately $64 \mathrm{mg} \mathrm{kg}^{-1}$ per day).

${ }^{1}$ Research Unit for Neurological Diseases, Instituto Mexicano del Seguro Social (IMSS), Mexico City, Mexico; ${ }^{2}$ Department of Experimental Surgery, Proyecto Camina A.C. Mexico City, Mexico; ${ }^{3}$ Department of Neurology, School of Veterinary Medicine, Universidad Autónoma del Estado de Mexico, Toluca, Mexico and ${ }^{4}$ Department of Biochemistry, Instituto Nacional de Rehabilitación, Mexico City, Mexico

Correspondence: Dr G Guizar-Sahagun, Research Unit for Neurological Diseases, Instituto Mexicano del Seguro Social (IMSS), Tlalpan 4430, Mexico City, DF 14050 Mexico, E-mail: guizarg@gmail.com

Received 9 February 2014; revised 31 March 2014; accepted 5 May 2014 

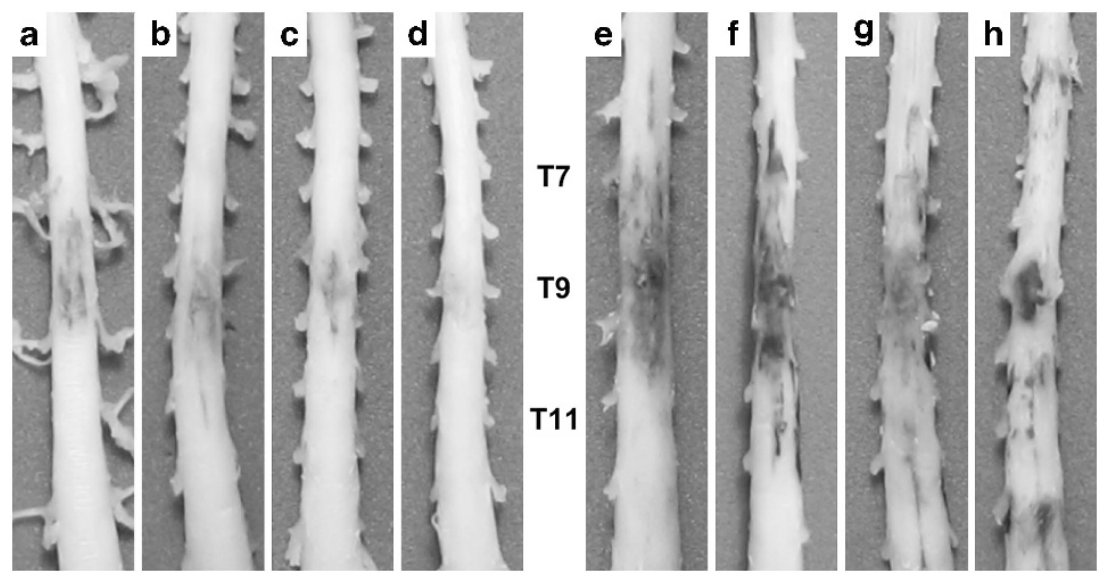

Figure 1 Spinal subarachnoid bleeding after graded cord injury. Macroscopic images show time course of blood deposition in the dorsal aspects of specimens from mildly (a-d) and severely (e-h) injured rats. Time post injury: (a and $\mathbf{e}) 1 \mathbf{h}$; (b and f) 1 day; (c and $\mathbf{g}$ ) 3 days; (d and $\mathbf{h}$ ) 7 days.
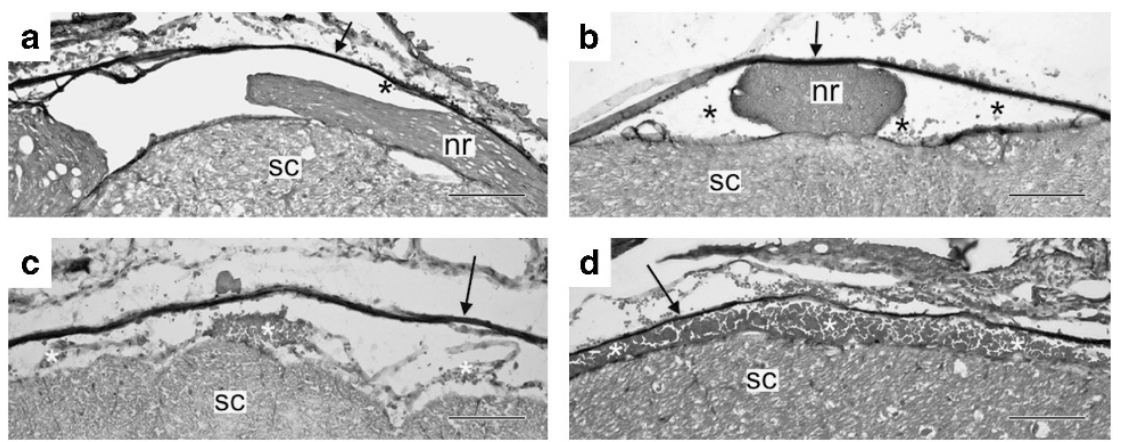

Figure 2 Spinal subarachnoid bleeding scoring. (a) 0, presence of only one erythrocyte $(*)$; (b) 1 , presence of free erythrocytes and very small clots $(*)$; (c) 2, medium-sized hematomas $\left({ }^{*}\right)$ occupying less than half of the SSAS; (d) 3, a large hematoma $\left(^{*}\right)$ filling the entire SSAS. Arrows, dural sac; sc, spinal cord; $\mathrm{nr}$, nerve root; Masson stained. Scale bar, $120 \mu \mathrm{m}$.

\section{Preparation of specimens}

Histology was carried out with the same specimens used for a previous report. ${ }^{5}$ Briefly, anesthetized rats were perfused by intracardiac puncture with saline solution, followed by $4 \%$ paraformaldehyde. Spine blocks were dissected out, post-fixed in the same fixative and then decalcified in $12 \%$ ethylenediaminetetraacetic acid. In total, $6-\mu \mathrm{m}$-thick transverse cryosections were obtained from each vertebral segment (T7-T12) at intervertebral discs. Sections were stained with the Masson method and propidium iodide for light and epifluorescent microscopy, respectively.

For macroscopic observations, rats were deeply anesthetized and perfused by intracardiac puncture with $200 \mathrm{ml}$ saline solution, followed by $500 \mathrm{ml} 10 \%$ buffered formaldehyde. Cords were dissected out keeping meninges intact, and were photographed.

\section{Video recording}

To view SSB immediately after mild and severe cord contusions, anesthetized/ laminectomized rats were placed in the NYU impactor. Video was recorded with an HD camcorder through a surgical microscope for about 1 min without interruption. Images were taken of the dorsal aspect of the dural sac before injury, at the moment of impact and for a few seconds thereafter.

\section{Analysis}

Using the relative grading frequencies of SSB, as determined by histological scorings ( $0-3)$, contingency tables were created and raw data analyzed with the $\chi^{2}$ test. Comparisons between mild and severe injuries were performed for the occurrence of SSB considering all areas examined (four circumferential areas into which all vertebral segments were divided, for all time points) or the condition of major bleeding (dorsal area of the injury epicenter, at $1 \mathrm{~h}$ and 1 day post injury). Progress, craniocaudal extension and circumferential location of bleeding were assessed and compared separately for mild and severe injuries; the vast differences of these parameters between both lesion groups merit no further statistical analysis. Differences were considered significant when $P<0.05$.

\section{Statement of ethics}

We certify that all applicable institutional and governmental regulations concerning the ethical use of animals were followed during the course of this research.

\section{RESULTS}

SSB started immediately after injury as shown in Supplementary videos 1 and 2. After severe injury, major bleeding arose from ruptured large vessels; small bleedings were observed at several points, apparently originating from pial and/or radicular vessels; single bleedings coalesced into subarachnoid hematomas (Supplementary video 1). For mild injuries, SSB appeared to arise from ruptured small vessels resulting in scattered minor bleedings, which did not pool to form hematomas (Supplementary video 2).

Macroscopically, SSB appeared more intense in severe vs mild injuries, at the epicenter and adjacent segments vs segments away from the site of injury, at dorsal vs lateral and ventral aspects, and at early vs late stages after injury (Figure 1).

To histologically score bleeding intensity, circumferential SSAS was divided into four areas: dorsal, lateral left, lateral right and ventral. Areas were defined by an X plane, which in most cases coincided with location of nerve roots. For $1 \mathrm{~h}$ through 3 days post injury, areas were graded depending on the amount of blood present as follows: 

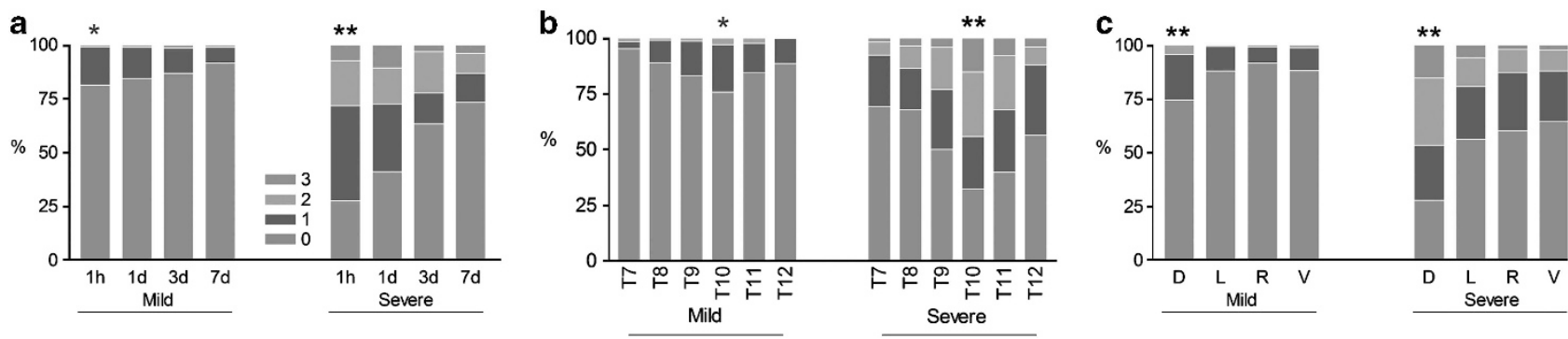

Figure 3 Progression and location of spinal subarachnoid bleeding. (a) Progression of extravasated blood for $1 \mathrm{~h}$ to 7 days post injury (includes scores of the four areas of each of the six vertebral segments studied); (b) craniocaudal extension of bleeding for each vertebral segment (includes the four areas of each segment, at all time points examined); (c) circumferential location of bleeding for each area: dorsal (D), lateral left (L), lateral right (R); ventral (V) (includes the six vertebral segments at all time points examined). Plots represent percentage of areas scored for bleeding on a scale from 0-3. Comparing data for mild and severe injuries separately, values were very significantly $\left(^{*}\right)$ or highly significantly $\left.{ }^{* *}\right)$ different.
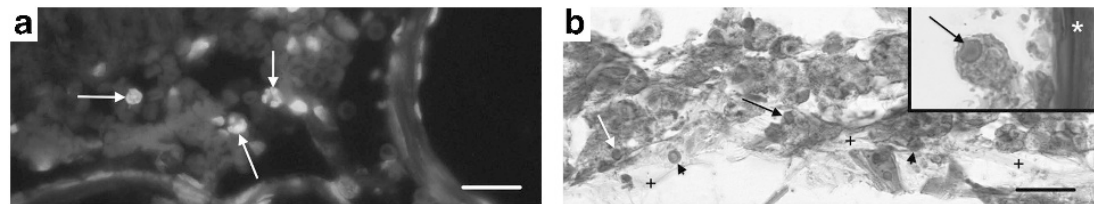

Figure 4 Inflammatory cells associated with extravasated blood in SSAS after severe injury. (a) Specimen taken 1 day post injury, showing polymorphonuclear leukocytes (arrows); (b) specimen obtained at 7 days post injury showing numerous macrophages with phagocytized erythrocytes (arrows). Free erythrocytes can also be seen (arrowheads). Both phagocytized and free erythrocytes are seen at various stages of disintegration. Within the SSAS, bands apparently of collagen $(+)$ are observed going in different directions. Insert shows a macrophage located in proximity to the dural sac $(*)$, containing a phagocytized erythrocyte (arrow). Stains: a, propidium iodide; b, Masson. Scale bar, $20 \mu \mathrm{m}$.

0 , absence or very few erythrocytes (no more than 5 per $\times 20$ field); 1 (minor), loose erythrocytes or very small clots found in SSAS; 2 (moderate), hematomas occupying less than half the area; 3 (major), large hematomas filling more than half the area (Figure 2). For specimens obtained 7 days post injury, scoring (0-3) depended on SSAS occupancy by blood at different stages of disintegration, macrophages with engulfed erythrocytes and collagen-like bands.

Regarding the incidence and extent of SSB, considering all areas examined, for all vertebral segments, at all experimental time points, occurrence of SSB after mild injury was observed in $15 \%$ of the areas ( $13 \%$ grade 1 and $2 \%$ grade 2 ), while the rest showed no bleeding at all; after severe injury, bleeding was observed in $48 \%$ of the areas ( $25 \%$ grade $1,17 \%$ grade 2 and $6 \%$ grade 3 ). The difference between SSB for mild and severe injuries was highly significant $(P<0.0001)$. Under conditions of major bleeding (dorsal area of the injury epicenter, at $1 \mathrm{~h}$ and 1 day post injury), rats with mild injury showed bleeding in $69 \%$ of the areas examined (63\% grade $1,6 \%$ grade 2 and none grade 3), while rats with severe injury showed bleeding in $100 \%$ of the areas (6\% grade $1,38 \%$ grade 2 and $56 \%$ grade 3 ). Bleeding for mild vs severe injuries was again highly significantly different $(P<0.0001)$.

In relation to the progression of SSB, extravasated blood within SSAS decreased significantly over time, most notably after severe injury going from $72 \%$ at $1 \mathrm{~h}$ to $27 \%$ at day $7(P<0.0001)$, and from $18 \%$ to $9 \%$, respectively, for mild injuries $(P=0.0106)$ (Figure $3 a)$.

With respect to the craniocaudal extension of SSB, scorings from vertebral segments T7-T12 showed the greatest hemorrhage to occur at the epicenter (T10), which was $68 \%$ for severe injuries and $24 \%$ for mild contusions; in both cases, bleeding decreased gradually from the site of injury. The least bleeding for both types of injuries was observed at T7 and was $31 \%$ for severe injuries $(P<0.0001)$ and $5 \%$ for mild lesions $(P=0.0031)$ (Figure $3 \mathrm{~b})$.

Regarding the circumferential areas of location of SSB for both types of injury severity, the greatest amount of bleeding was located dorsally: $72 \%$ for severe injuries and $26 \%$ for mild lesions. The least amount of bleeding was observed in ventral areas for severe injuries $(35 \%, P<0.0001)$ and in lateral right areas for mild contusions $(8 \%, P<0.0001)$ (Figure 3c).

Inflammatory cells associated with SSB at $1 \mathrm{~h}, 1$ day and 3 days after injury were polymorphonuclear leukocytes; by day 7 after injury, inflammatory cells observed were macrophages (Figure 4).

\section{DISCUSSION}

To the best of our knowledge, this is the first observational systematic report on SSB associated to cord injury, with potential pathophysiological and therapeutic implications.

Our results show that occurrence and extent of SSB associated to SCI depend primarily on the severity of the injury. The differences in the incidence and extent of bleeding between mild and severe injuries are highly significant both when considering all areas examined in the study or only those areas of heavier bleeding.

Our findings are in agreement with those reported earlier on the extent of SSB as a function of the intensity of injury in experimental graded SCI, ${ }^{4,5}$ although here we further describe the patterns of SSB associated to the intensity of SCI.

Spinal SSB occurs rarely from causes different than SCI. It has been associated to iatrogenic events such as lumbar puncture or minor vertebral trauma, both in patients with defective coagulation, characterized by an acute onset of symptoms, frequently leading to rapid neurological deterioration. ${ }^{8}$

\section{Patterns of SSB}

SSB starts with the rupture of vessels of different caliber in SSAS at the moment of contusion; the more severe the blow, the greater the extent of vessel rupture (Supplementary videos 1 and 2).

The greatest amount of bleeding occurs in the dorsal portion of the epicenter, precisely at the site of impact. From there, blood diffuses 
through SSAS in both craniocaudal and circumferential directions, gradually decreasing as it moves away from the lesion epicenter.

For both mild and severe injuries, most SSB appears within the first hour after trauma. We can assume that, over time, part of the extravasated blood (loose erythrocytes and small clots) is gradually cleared by the CSF, leaving behind hematomas, which are too big to be washed out in this manner. In addition, other routes for subarachnoid blood clearing might involve hemolysis and removal by phagocytic cells, mainly macrophages, seen 7 days post injury (see Figure $4 b$ ).

\section{Possible implications of SSB on SCI outcome}

Brain subarachnoid bleeding in humans and experimental animals, resulting from events such as ruptured aneurysms or brain injury, triggers inter-related pathomechanisms partially attributable to the release of toxic byproducts of hemoglobin degradation, ${ }^{10-12}$ leading to irreversible brain damage and even death. Delayed ischemic neurological deficit is recognized as the most prominent complication of brain subarachnoid bleeding, ${ }^{10,12}$ and has been associated to several mechanisms including vasospasms, ${ }^{10}$ failure of blood flow autoregulation, ${ }^{7}$ disruption of the blood-brain barrier, ${ }^{12}$ edema, ${ }^{12}$ microthrombosis, ${ }^{13}$ inflammation ${ }^{11}$ and oxidative stress, ${ }^{10}$ among others. Conditions such as these can in turn lead to alterations in neuronal signal processing and transmission, ${ }^{12}$ infarctions ${ }^{6}$ and gliosis. ${ }^{14}$

Similar mechanisms of lesion occur after SCI. ${ }^{1,2}$ It is therefore reasonable to speculate that the extravasated blood in SSAS (described here) may, in fact, contribute to the pathophysiology of acute SCI.

In addition, spinal subarachnoid hematomas might contribute to the development of posttraumatic arachnoiditis. Our observations on day 7 post injury, show fibrous stretches of collagen within these bloody structures that appear to be in the process of dissolution. These observations are in agreement with the findings of Sajanti et al. ${ }^{15}$ who reported an increase in CSF concentration of procollagen propeptides in patients with recent subarachnoid hemorrhaging. In fact, since the very early studies of Bagley ${ }^{16}$ extravasated subarachnoid blood has been causally linked to chronic spinal arachnoiditis. ${ }^{15}$ Moreover, under the same experimental conditions used here, cord tethering occurred at the chronic stage in animals with severe injury, dorsally to the epicenter precisely at the site of maximum SSB. ${ }^{5}$ Arachnoiditis, in turn, may lead to syringomyelia by alterations in dynamics of CSF flow ${ }^{17}$ and spinal neuropathic pain due to nerve root entrapment. ${ }^{18}$

\section{Study limitations}

Being an observational study based on morphological analyses, our data do not provide information on mechanisms by which SSB could contribute to cord damage after SCI. We believe, however, that our report is a solid basis to formulate new hypotheses to test for pathological risks of such bleeding and eventual benefits from its control.

\section{CONCLUSIONS}

SSB occurs after experimental blunt graded SCI and depends on the severity of injury being significantly greater after severe contusions compared with mild lesions. The greatest amount of blood in SSAS regardless of the intensity of trauma occurs early after injury and is found dorsally at the epicenter and decreases gradually, over time, away from this site.

The detailed description offered here on SSB patterns occurring after graded SCI is a starting point to achieve a better understanding of extramedullary events related to cord injury and may lead to the design of novel, more effective therapeutic strategies.

\section{DATA ARCHIVING}

There were no data to deposit.

\section{CONFLICT OF INTEREST}

The authors declare no conflict of interest.

\section{ACKNOWLEDGEMENTS}

We thank Francisco Márquez and Álvaro Corona-Juárez for their technical assistance. Grants were from the Instituto Mexicano del Seguro Social (C2007/ 037) and the Consejo Nacional de Ciencia y Tecnología (104771 I0110/194/09).

1 Guizar-Sahagun G, Rodriguez-Balderas CA, Franco-Bourland RE, Martinez-Cruz A, Grijalva I, Ibarra A et al. Lack of neuroprotection with pharmacological pretreatment in a paradigm for anticipated spinal cord lesions. Spinal Cord 2009; 47: 156-160.

2 Stahel PF, VanderHeiden T, Finn MA. Management strategies for acute spinal cord injury: current options and future perspectives. Curr Opin Crit Care 2012; 18: 651-660.

3 Yablon IG, Ordia J, Mortara R, Reed J, Spatz E. Acute ascending myelopathy of the spine. Spine (Phila Pa 1976) 1989; 14: 1084-1089.

4 Koenig G, Dohrmann GJ. Histopathological variability in 'standardised' spinal cord trauma. J Neurol Neurosurg Psychiatry 1977; 40: 1203-1210.

5 Reyes-Alva HJ, Franco-Bourland RE, Martinez-Cruz A, Grijalva I, Madrazo I, Guizar-Sahagun G. Spatial and temporal morphological changes in the subarachnoid space after graded spinal cord contusion in the rat. J Neurotrauma 2013; 30: 1084-1091.

6 Schatlo B, Dreier JP, Glasker S, Fathi AR, Moncrief T, Oldfield EH et al. Report of selective cortical infarcts in the primate clot model of vasospasm after subarachnoid hemorrhage. Neurosurgery 2010; 67: 721-728.

7 Budohoski KP, Czosnyka M, Kirkpatrick PJ, Smielewski P, Steiner LA, Pickard JD. Clinical relevance of cerebral autoregulation following subarachnoid haemorrhage. Nat Rev Neurol 2013; 9: 152-163.

8 Domenicucci M, Ramieri A, Paolini S, Russo N, Occhiogrosso G, Di Biasi C et al. Spinal subarachnoid hematomas: our experience and literature review. Acta Neurochir (Wien) 2005; 147: 741-750.

9 Metz GA, Curt A, van de Meent H, Klusman I, Schwab ME, Dietz V. Validation of the weight-drop contusion model in rats: a comparative study of human spinal cord injury. J Neurotrauma 2000; 17: 1-17.

10 Echigo R, Shimohata N, Karatsu K, Yano F, Kayasuga-Kariya Y, Fujisawa A et al. Trehalose treatment suppresses inflammation, oxidative stress, and vasospasm induced by experimental subarachnoid hemorrhage. J Trans/ Med 2012; 10: 80.

11 Greenhalgh AD, Brough D, Robinson EM, Girard S, Rothwell NJ, Allan SM. Interleukin-1 receptor antagonist is beneficial after subarachnoid haemorrhage in rat by blocking haem-driven inflammatory pathology. Dis Model Mech 2012; 5: 823-833.

12 Kamp MA, Dibue M, Etminan N, Steiger HJ, Schneider T, Hanggi D. Evidence for direct impairment of neuronal function by subarachnoid metabolites following SAH. Acta Neurochir (Wien) 2013; 155: 255-260.

13 Sabri M, Ai J, Lakovic K, D'Abbondanza J, Ilodigwe D, Macdonald RL. Mechanisms of microthrombi formation after experimental subarachnoid hemorrhage. Neuroscience 2012; 224: 26-37.

14 Murakami K, Koide M, Dumont TM, Russell SR, Tranmer BI, Wellman GC. Subarachnoid hemorrhage induces gliosis and increased expression of the proinflammatory cytokine high mobility group box 1 protein. Trans/ Stroke Res 2011; 2: 72-79.

15 Sajanti J, Heikkinen E, Majamaa K. Transient increase in procollagen propeptides in the CSF after subarachnoid hemorrhage. Neurology 2000; 55: 359-363.

16 Bagley C. Blood in the cerebrospinal fluid: resultant functional and organic alterations in the central nervous system A. Experimental data. Arch Surg 1928; 17: 18-38.

17 Seki T, Fehlings MG. Mechanistic insights into posttraumatic syringomyelia based on a novel in vivo animal model. Laboratory investigation. J Neurosurg Spine 2008; 8: 365-375.

18 Gross R, Hamel O, Robert R, Perrouin-Verbe B. Perilesional myeloradiculopathy with tethered cord in post-traumatic spinal cord injury. Spinal Cord 2013; 51: 369-374. 